\title{
Necrotizing fasciitis following primary peritonitis caused by Streptococcus pyogenes with covS mutation in a healthy woman: a case report
}

\author{
Masashi Inoue* (D), Eisuke Kako, Rie Kinugasa, Fumiaki Sano, Hironobu Iguchi and Kazuya Sobue
}

\begin{abstract}
Background: Primary peritonitis due to Streptococcus pyogenes (S. pyogenes) is uncommon in patients without comorbid conditions such as immunosuppression, nephritic disease, or liver cirrhosis. Furthermore, it does not cause another infection at the same time in a healthy person. However, several S. pyogenes mutants have been reported, and some of them exhibit strong virulence. Mutation of the control of virulence (cov) S gene of Streptococcus enhances bacterium survival by repressing negative regulators of virulence, which causes bacterial invasion of aseptic tissues, such as the parenteral space. We report a case of primary peritonitis and subsequent necrotizing fasciitis by the same S. pyogenes species with mutated covS in a previously healthy woman.
\end{abstract}

Case presentation: We present the case of a 55 -year-old woman admitted to the hospital due to abdominal pain and nausea. She was treated for peritonitis. A few days later, she became hypotensive and tachycardic and was transferred to the intensive care unit (ICU) for the treatment of septic shock with primary peritonitis. On the second day of her ICU stay, both of her forearms developed swelling and redness around the peripheral injection site. The patient had developed necrotizing fasciitis. Since her skin symptoms spread rapidly, urgent debridement was performed. Her condition improved with antibiotic treatment and multiple episodes of debridement. S. pyogenes was detected in cultures of the patient's blood, ascites, and skin. The identified strain was emm89 genotype and had a genetic mutation of covs.

Conclusions: S. pyogenes with covS mutation may spread from a portal, such as the upper respiratory tract or digestive system, to all organs immediately, causing septic shock. Infection with S. pyogenes with mutated genes should be considered in the differential diagnosis of gastrointestinal symptoms, even in a previously healthy patient.

Keywords: Primary peritonitis, Necrotizing fasciitis, Streptococcus pyogenes, CovS mutation

\section{Background}

Primary peritonitis is an acute infection of the peritoneum without an obvious causative source; it is uncommon in the absence of comorbid conditions, including immunosuppression, nephritic disease, or liver cirrhosis, particularly in healthy individuals [1]. Primary peritonitis can be caused by infection with Enterobacteriaceae (e.g., Escherichia coli, Klebsiella spp.), Streptococcus pneumonia,

\footnotetext{
*Correspondence: m.inoue.pc@gmail.com

Department of Anesthesiology and Intensive Care Medicine, Nagoya City University Graduate School of Medical Sciences, 1-Kawasumi, Mizuho-cho, Mizuho-ku, Nagoya 467-8601, Japan
}

and Enterococcus spp.; however, it is rarely associated with Streptococcus pyogenes, which usually causes pharyngitis, erysipelas, and tonsillitis. Necrotizing fasciitis is a severe infection due to S. pyogenes, which has a high mortality. This infection generally occurs in the extremities around the gate of bacterial invasion in the skin such as trauma and surgical wound. It is very rare to develop necrotizing fasciitis secondary to primary peritonitis by the same bacteria species. Recent studies have suggested that $S$. pyogenes carrying mutations in covS gene can cause severe invasive infections. In this report, we describe the investigation and management of a 55-year-old woman with 
primary peritonitis and necrotizing fasciitis caused by $S$. pyogenes with a mutated covS.

\section{Case presentation}

A previously healthy 55-year-old woman entered a general ward complaining of lower abdominal pain and nausea. She was diagnosed with gastroenteritis and received fluid rehydration therapy with $1500 \mathrm{~mL}$ of hypotonic electrolyte solution per day, and intravenous acetaminophen was used for abdominal pain. Her nausea was improved after admission, so she did not take antiemetics. Her vital signs included a heart rate of $103 / \mathrm{min}$, blood pressure of $104 / 67 \mathrm{mmHg}$, oxygen saturation of $97 \%$ while breathing ambient air, and temperature of $39.7^{\circ} \mathrm{C}$. Laboratory data showed a white blood cell count of $29.5 \times 10^{9} / \mathrm{L}$ and C-reactive protein of $19.3 \mathrm{mg} / \mathrm{dL}$. An abdominal computed tomography (CT) examination showed a thickened wall of the small intestine but no ascites (Fig. 1). She had no travel history and had not taken any medications or consumed any perishables. Any bacterial culture examination was not performed until ICU admission because infectious disease was not anticipated. Despite fluid rehydration therapy, her general condition worsened. On day 6 , she entered the ICU because of disturbance of consciousness and respiratory failure. Before ICU admission, her vital signs were as follows: heart rate, $135 / \mathrm{min}$; systolic blood pressure, 60 $\mathrm{mmHg}$; and respiratory rate, 50 breaths/min. Laboratory data showed a white blood cell count of $10.6 \times 10^{9} / \mathrm{L}$ and elevated C-reactive protein of $34.2 \mathrm{mg} / \mathrm{dL}$. An abdominal CT showed intestinal edema and ascites on the liver and Douglas's fossa (Fig. 2). Her limbs were cold, and both forearms were swollen because of the leakage of the peripheral infusion. Furthermore, she had respiratory distress. Septic shock was suspected, and she was treated with fluid resuscitation, empirical antibiotics (meropenem, linezolid, and micafungin), and a vasopressor. On ICU day 2, her arms became swollen and red (Fig. 3). $\mathrm{CT}$ of the upper limbs showed edema between the muscles, suggesting necrotizing fasciitis (Fig. 4). Therefore, emergency skin incision and debridement were performed. A pathological examination showed intense polymorphonuclear cell infiltration with diffuse connective tissue necrosis, compatible with a diagnosis of necrotizing fasciitis. Renal replacement therapy was also initiated because of acute kidney injury. Serum creatinine had increased from 0.72 to $3.03 \mathrm{mg} / \mathrm{dL}$ (stage 3 based on the KDIGO classification). Surprisingly, S. pyogenes was detected from cultures of ascitic fluid, which were taken before sepsis developed, from blood and skin. The empirical antibiotics were switched to penicillin G, linezolid, and clindamycin (linezolid was discontinued on ICU day 8). On ICU day 3, $1420 \mathrm{~mL}$ of ascites was drained and the patient underwent second debridement of the upper limbs, because the inflammation had extended over the shoulders. Her condition improved, and she overcame the septic shock on ICU day 6. On ICU day 9, Pseudomonas aeruginosa and Candida parapsilosis were found in a skin culture. Therefore, the antibiotics were switched to ciprofloxacin and micafungin. Flap angioplasties of both forearms were performed on ICU day 22. The pedicle flaps were harvested from each intact area of the arms and were placed to defect sites of the respective limbs, then additional skin grafting was

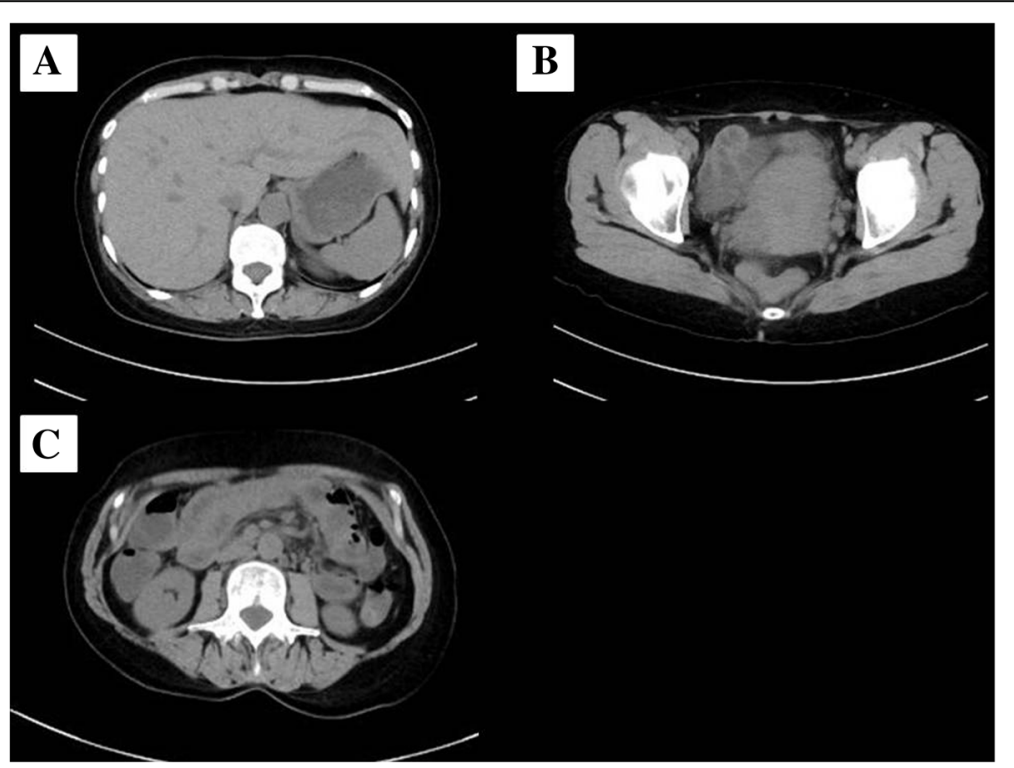

Fig. 1 Abdominal computed tomography examination on admission. Abdominal computed tomography examination on admission showed no ascites $(\mathbf{a}, \mathbf{b})$ and a thickened wall throughout the entire small intestine (c) 


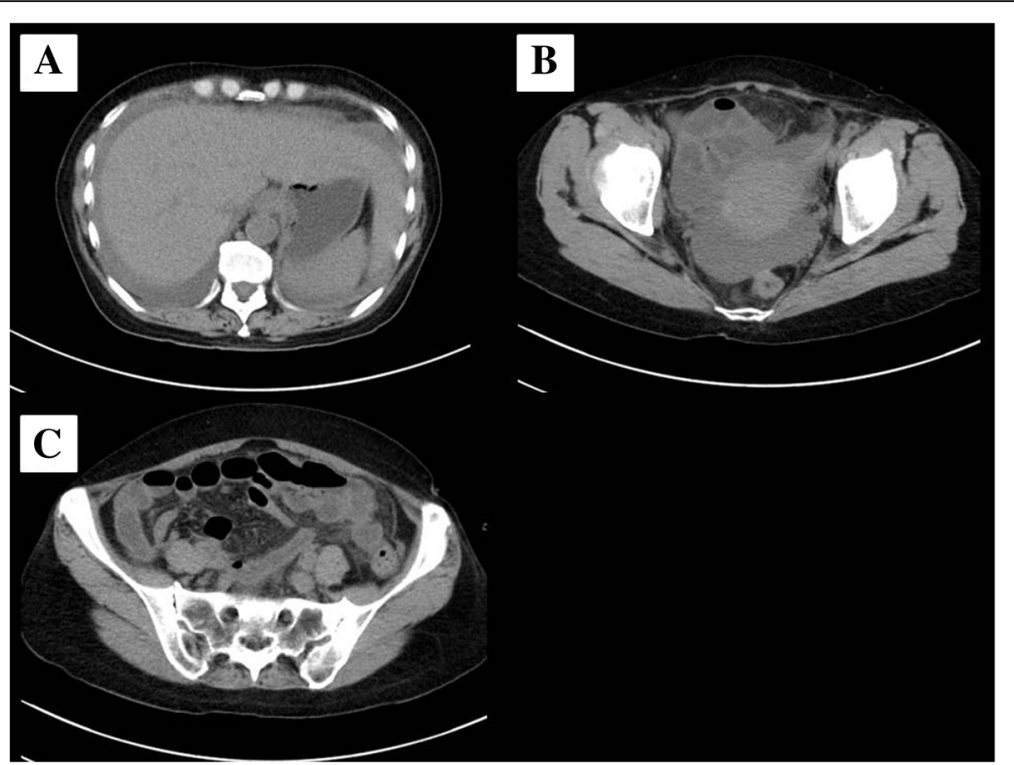

Fig. 2 Computed tomography of the abdomen. Computed tomography of the abdomen detected ascites on the liver (a) and Douglas's fossa (b). CT imaging also showed intestinal edema (c)

performed on ICU day 36. The patient was transferred from the ICU to the general ward on day 62 (ICU day 57). She was discharged to a regional medical support hospital on day 110 .

A genetic examination of the S. pyogenes harvested from the skin and blood cultures showed that the strain had a mutation in the covS gene.

\section{Conclusions}

There are some reports of peritonitis caused by S. pyogenes infection in healthy women [2-7]. The predominance of women among $S$. pyogenes peritonitis patients might implicate ascending infections of the genitourinary tract, since S. pyogenes are often part of the vaginal flora [8]. According to a systematic review of primary

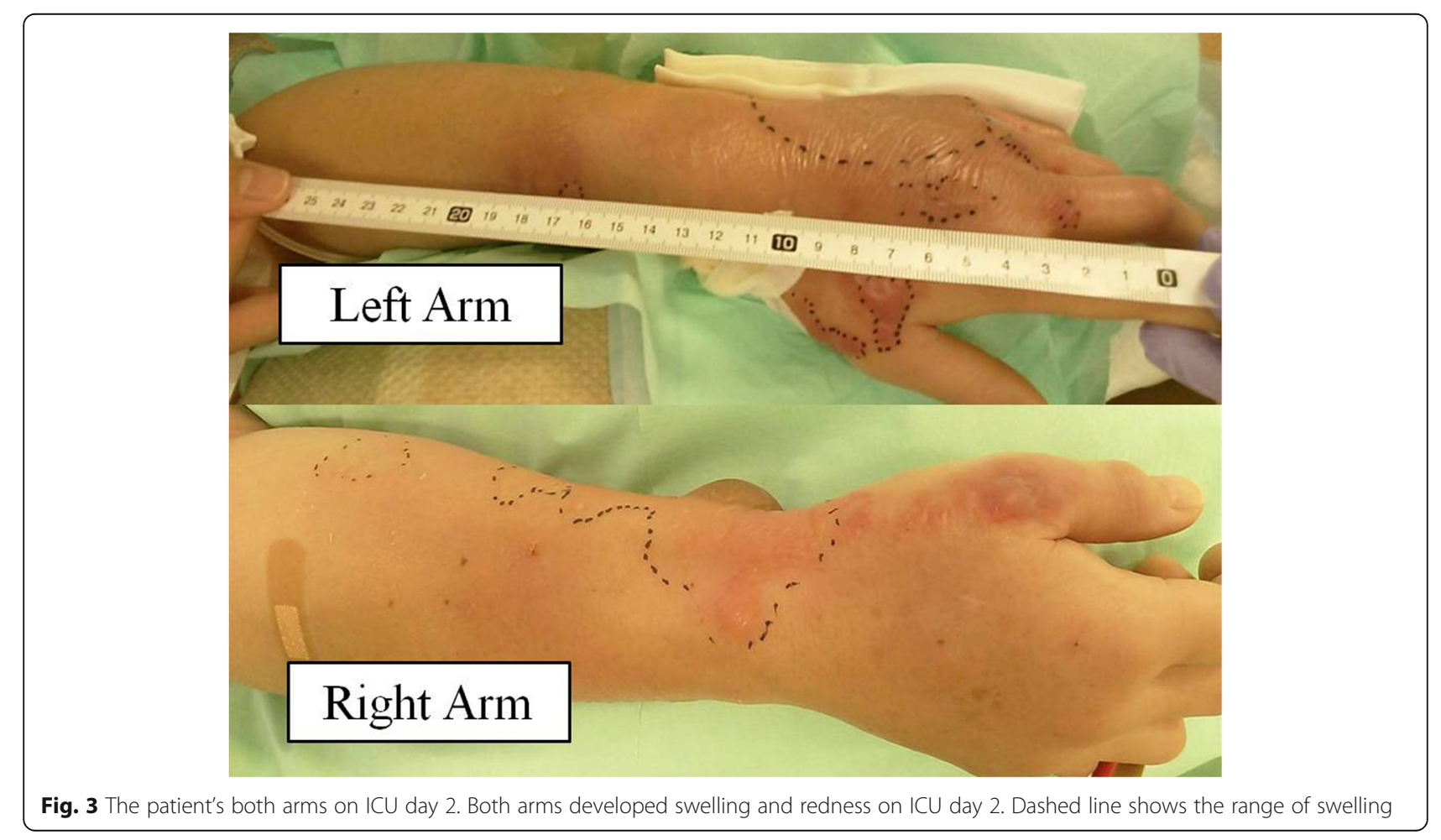



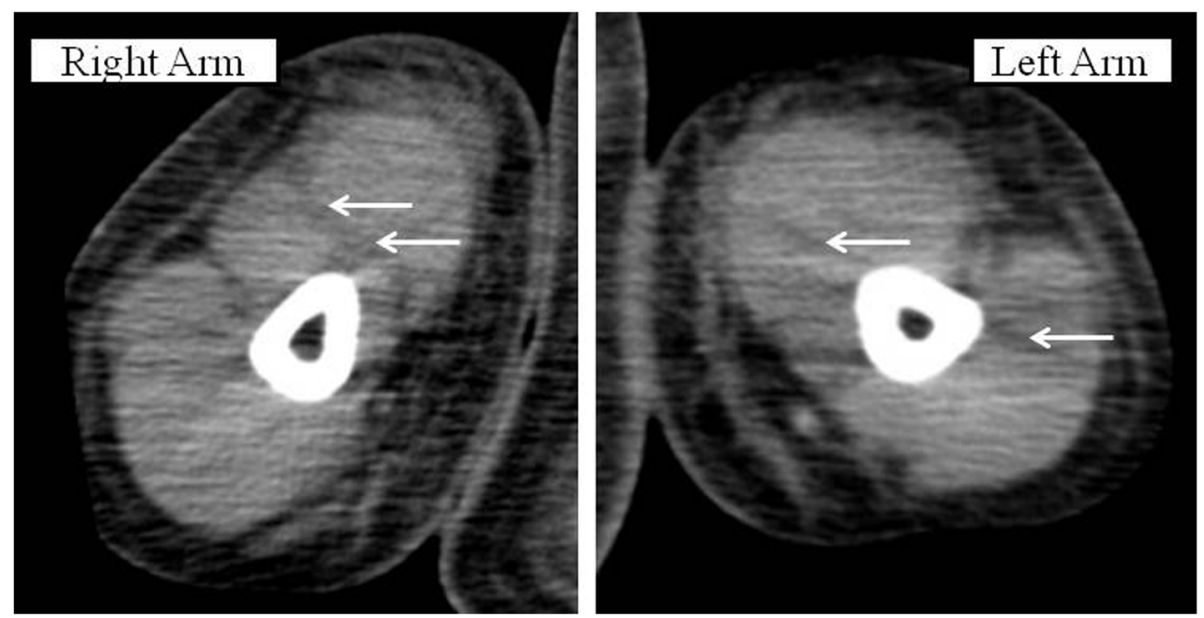

Fig. 4 Computed tomography images of the forearms. Non-contrast CT images show edema between the muscles (white arrows)

peritonitis caused by streptococcus, $16 \%$ of patients developed streptococcal toxic shock syndrome (STSS) from an ascending infection originating from the vagina, 9\% of patients developed it from a droplet infection, $6 \%$ of patients developed it from pharyngitis, and $69 \%$ of patients developed it via an unknown route [9]. In our case, we could not determine the primary site of infection; we should have collected a vaginal culture to confirm whether the infection originated in the vaginal tract. For the same reason, we should have collected a pharynx culture. The diagnosis of peritonitis was based on the presence of purulent ascites cultures with increased lactate dehydrogenase (LDH) and white blood cells (WBC) and CT findings of increased intensity within the peritoneum and smooth, uniform thickening of the small intestine wall.

Necrotizing fasciitis is an infection of the deeper subcutaneous tissue and fascia that is characterized by rapidly spreading necrosis of the skin. The successful management of necrotizing fasciitis is dependent on early recognition, but classical signs of a necrotizing process are not initially apparent. If the patients have a defined portal of entry, such as a surgical incision, burn, insect bite, or varicella lesion, the skin around will be red with severe pain and swelling. However, in the 50\% of patients who develop necrotizing fasciitis without a defined portal of entry, the infection begins deep in the tissues, frequently at the site of a hematoma, muscle strain, or traumatic joint injury [1]. There are some studies of necrotizing fasciitis associated with drug injection $[10,11]$. One study found that $54 \%$ of drug users with necrotizing fasciitis developed the infection at an injection site [10]. The same study showed that $S$. pyogenes was the third most frequently detected gram-positive aerobe, following Staphylococcus aureus and viridans streptococci. Another study revealed that patients with a necrotizing fasciitis after injection or infiltration therapy have a poor prognosis in terms of survival and amputation rate [11]. In our case, redness and swelling spread from peripheral infusion cannulation sites on both forearms. Thus, the S. pyogenes infection might have originated from the injection sites. However, it is considered that the necrotizing fasciitis was spread from primary peritonitis via bloodstream infection which caused septic shock at ICU admission. The reason is that S. pyogenes species which had the same sensitivity to antibiotics were detected from ascites, blood, and skins in both arms. Then, as of ICU admission, there were no inflammation signs in the limbs even though peritonitis and sepsis had already occurred. Furthermore, she developed necrotizing fasciitis concurrently in both arms which were apart from each other. It seems questionable if the bacteria involved in the fasciitis invaded from the skin.

Additionally, this $S$. pyogenes lineage comprised emm89type strains and carried a mutation in covS gene. This group produces many surface-bound and extracellular virulence factors that contribute to pathogenesis in complex ways. CovS/CovR is a key two-component regulatory system of gene transcription in S. pyogenes [12-14]. CovS/CovR regulates the expression of $15 \%$ of the genes of $S$. pyogenes [15, $16]$, including streptococcal pyrogenic exotoxin B (SpeB). Because the expression of active $\mathrm{SpeB}$ causes the degradation of secreted GAS (group A streptococci) proteins, including several virulence factors, reduced SpeB expression in covS mutants confers strong virulence on S. pyogenes $[17,18]$. Recent studies have also described an inverse relationship between disease severity and the degree of a cysteine protease, SpeB $[12,13]$. Moreover, a previous report has suggested that mutated covS plays important roles in the in vivo dissemination of $S$. pyogenes in humans from the upper respiratory tract to aseptic tissues, such as blood and cerebrospinal fluid [19], and some studies have described the significance of 
covS mutations in the pathogenesis of STSS [20, 21]. In this case, it was considered that this covS mutation contributed to the development of peritonitis in a previously healthy patient.

In our case, the $S$. pyogenes was sensitive to penicillin G. Therefore, we used penicillin G and clindamycin as definitive therapies. We also used linezolid until the pathogen was identified because the possibility of community-acquired methicillin-resistant Staphylococcus aureus (MRSA) involvement could not be ruled out. The guidelines of the Infectious Disease Society of America recommend the use of penicillin plus clindamycin for the treatment of documented streptococcal necrotizing fasciitis [22]. This recommendation is based on the finding that all strains of $S$. pyogenes are sensitive to penicillin and that clindamycin suppresses $S$. pyogenes exotoxin and $M$ protein production. $M$ protein is an important molecule for adhering to host cells; furthermore, it has anti-opsonin activity, which is associated with resistance to immunity. Moreover, the guidelines show that empirical treatment of polymicrobial necrotizing fasciitis should include agents that are effective against both aerobes, including MRSA, and anaerobes.

In summary, we report a rare case of secondary necrotizing fasciitis following primary peritonitis with a S. pyogenes infection in a previously healthy woman. $S$. pyogenes primary peritonitis should be considered in the differential diagnosis of gastrointestinal symptoms, even in healthy women. In these cases, it is important to detect the pathogen underlying peritonitis through abdominocentesis or a diagnostic laparotomy. Subsequently, antibiotic therapy should be initiated promptly. Because $S$. pyogenes with mutated covS may spread immediately from the upper respiratory tract, the vagina, or a peripheral injection site to the organs, rapid diagnosis and treatment are required. Furthermore, it is important to avoid unnecessary catheter placement to prevent catheter-related blood stream infection.

\section{Abbreviations}

Cov: Control of virulence; CT: Computed tomography; GAS: Group A streptococci; ICU: Intensive care unit; MRSA: Methicillin-resistant Staphylococcus aureus; S. pyogenes: Streptococcus pyogenes; SpeB: Streptococcal pyrogenic exotoxin B; STSS: Streptococcal toxic shock syndrome

\section{Acknowledgements}

Not applicable.

\section{Funding}

Our research was not funded by any agencies.

\section{Availability of data and materials}

Data sharing is not applicable to this article as no datasets were generated or analyzed during the current study.

\section{Authors' contributions}

Ml collected and interpreted the patient's bacteriologic data and was a major contributor to writing the manuscript. EK, RK, FS, HI, and KS gave important advice on the manuscript in terms of literature considerations. All the authors read and approved the final manuscript.

\section{Ethics approval and consent to participate}

We obtained the patient's consent to participate.

Consent for publication

We obtained the patient's consent using our institutional consent form.

\section{Competing interests}

The authors declare that they have no competing interests.

\section{Publisher's Note}

Springer Nature remains neutral with regard to jurisdictional claims in published maps and institutional affiliations.

Received: 12 February 2019 Accepted: 15 April 2019

Published online: 27 April 2019

References

1. Stevens DL, Bryant AE. Severe group a streptococcal infections. In: Streptococcus pyogenes: basic biology to clinical manifestations. University of Oklahoma Health Sciences Center. 2016. https://www.ncbi.nlm.nih.gov/ books/NBK333425/. Accessed 17 Dec 2018.

2. Holden R, Wilmer A, Kollmann T. Primary peritonitis due to group A Streptococcus in a previously healthy pediatric patient. Can J Infect Dis Med Microbiol. 2012;23:69-70.

3. Park JY, Youn MS, Son JS, Lee MS, Jung MH. Unusual primary peritonitis due to Streptococcus pyogenes in a young healthy woman. J Korean Med Sci. 2012;27:553-5.

4. Tilanus AMR, de Geus HRH, Rijnders BJA, Dwarkasing RS, van der Hoven B, Bakker J. Severe group A streptococcal toxic shock syndrome presenting as primary peritonitis: a case report and brief review of the literature. Int J Infect Dis. 2010;14(Suppl 3):208-12.

5. Yokoyama M, Oyama F, Ito A, Yokota M, Matsukura D, Tsutsumi S, Kasai T, Nitobe Y, Morikawa A, Ozaki T, Yokoyama Y. Streptococcal toxic shock syndrome: life saving role of peritoneal lavage and drainage. Clin Med Insights Case Rep. 2016;9:75-7.

6. Malota M, Felbinger TW, Ruppert R, Nüssler NC. Group A Streptococci: a rare and often misdiagnosed cause of spontaneous bacterial peritonitis in adults. Int J Surg Case Rep. 2015;6:251-5.

7. Haap M, Haas CS, Teichmann R, Horger M, Raible A, Lamprecht G. Mystery or misery? Primary group a streptococcal peritonitis in women: case report. Am J Crit Care. 2010;19:454-8.

8. Monneuse O, Tissot E, Gruner L, Michaillard Kaempf F, Allaouchiche B, Etienne J, Barth X. Diagnosis and treatment of spontaneous group A streptococcal peritonitis. Br J Surg. 2010;97:104-8.

9. Westwood DA, Roberts RH. Management of primary group A streptococcal peritonitis: a systematic review. Surg Infect. 2013;14:171-6.

10. Chen JL, Fullerton KE, Flynn NM. Necrotizing fasciitis associated with injection drug use. Clin Infect Dis. 2001;33:6-15.

11. Friederichs J, Torka S, Militz M, Bühren V, Hungerer S. Necrotizing soft tissue infections after injection therapy: higher mortality and worse outcome compared to other entry mechanisms. J Inf Secur. 2015;71:312-6.

12. Kansal RG, Datta V, Aziz RK, Abdeltawab NF, Rowe S, Kotb M. Dissection of the molecular basis for hypervirulence of an in vivo-selected phenotype of the widely disseminated M1T1 strain of group a Streptococcus bacteria. J Infect Dis. 2010;201:855-65.

13. Aziz RK, Pabst MJ, Jeng A, Kansal R, Low DE, Nizet V, Kotb M. Invasive M1T1 group A Streptococcus undergoes a phase-shift in vivo to prevent proteolytic degradation of multiple virulence factors by SpeB. Mol Microbiol. 2004;51:123-34

14. Cole JN, Barnett TC, Nizet V, Walker MJ. Molecular insight into invasive group A streptococcal disease. Nat Rev Microbiol. 2011;9:724-36.

15. Graham MR, Smoot LM, Migliaccio CA, Virtaneva K, Sturdevant DE, Porcella SF, Federle MJ, Adams GJ, Scott JR, Musser JM. Virulence control in group A Streptococcus by a two-component gene regulatory system: global expression profiling and in vivo infection modeling. Proc Natl Acad Sci U S A. $2002 ; 99: 13855-60$ 
16. Dalton TL, Collins JT, Barnett TC, Scott JR. RsCA, a member of the MDR1 family of transporters, is repressed by CovR and required for growth of Streptococcus pyogenes under heat stress. J Bacteriol. 2006;188:77-85.

17. Ato M, Ikebe T, Kawabata H, Takemori T, Watanabe H. Incompetence of neutrophils to invasive group A streptococcus is attributed to induction of plural virulence factors by dysfunction of a regulator. PLoS One. 2008;3: e3455.

18. Ikebe $T$, Ato M, Matsumura T, Hasegawa $H$, Sata T, Kobayashi $K$, Watanabe $H$. Highly frequent mutations in negative regulators of multiple virulence genes in group A streptococcal toxic shock syndrome isolates. PLoS Pathog. 2010;6:e1000832.

19. Masuno K, Okada R, Zhang Y, Isaka M, Tatsuno I, Shibata S, Hasegawa T. Simultaneous isolation of emm89-type streptococcus pyogenes strains with a wild-type or mutated covS gene from a single streptococcal toxic shock syndrome patient. J Med Microbiol. 2014;63:504-7.

20. Garcia AF, Abe LM, Erdem G, Cortez CL, Kurahara D, Yamaga K. An insert in the covS gene distinguishes a pharyngeal and a blood isolate of Streptococcus pyogenes found in the same individual. Microbiology. 2010; 156:3085-95.

21. Tatsuno I, Okada R, Zhang Y, Isaka M, Hasegawa T. Partial loss of CovS function in Streptococcus pyogenes causes severe invasive disease. BMC Res Notes. 2013:6:126.

22. Stevens DL, Bisno AL, Chambers HF, Dellinger EP, Goldstein EJ, Gorbach SL, Hirschmann JV, Kaplan SL, Montoya JG, Wade JC. Practice guidelines for the diagnosis and management of skin and soft tissue infections: 2014 update by the Infectious Diseases Society of America. Clin Infect Dis. 2014. https:// doi.org/10.1093/cid/ciu444.

\section{Submit your manuscript to a SpringerOpen ${ }^{\circ}$ journal and benefit from:}

- Convenient online submission

- Rigorous peer review

- Open access: articles freely available online

- High visibility within the field

- Retaining the copyright to your article

Submit your next manuscript at $\boldsymbol{\nabla}$ springeropen.com 